\title{
Eine Frage der Perspektive: DER BALKANKRIEG IN DER DEUTSCHEN LITERATUR
}

\author{
BORIS PREVIŠIĆ
}

Wie vielen Begriffen ergeht es auch dem hier im Titel gewählten 'Balkan', wenn er seine Wirkungsmacht richtig entfaltet: Was ursprünglich ein Gebirge in Bulgarien bezeichnete und immer noch bezeichnet, steht heute für eine Problemregion, die sich vom eigentlichen Ort schon lange losgekoppelt und für ein Trauma Europas steht, deren Verarbeitung erst in der jüngsten Literatur einsetzt: Es geht um die Ende des 20. Jahrhunderts in Europa nicht mehr für möglich gehaltenen kriegerischen Auseinandersetzungen im ehemaligen Jugoslawien. 'Balkan' wird metaphorisch gebraucht und hier mit Bedacht auch so verwendet, denn - so fragt Peter Handke - "welch erwachsener Leser verbindet heutzutage überhaupt noch etwas Wirkliches mit solch einem Wort?"1 Was mit dem Prädikat 'balkanesisch' (ebd., S. 17) versehen wird, entspricht einem kulturellen Konstrukt, das sich in einem Abgrenzungsprozess ausformte, welcher in einem ersten Schub während der beiden Balkankriege 1912 und 1913 entstand, aber erst Ende der 80er Jahren in der Diskussion um 'Mitteleuropa' fortgesetzt wurde und sich während dem Zerfall Jugoslawiens nochmals verstärkte. Die bulgarischstämmige Historikerin Maria Todorova geht in ihrer bewusst gewählt provozierend-ironischen Schreibweise hingegen wieder so weit, den jüngsten Kriegen der 90er Jahre die Zuschreibung 'Balkankonflikte' abzusprechen, da alle übrigen Länder der Balkanhalbinsel wie Albanien, Bulgarien, Griechenland und Rumänien im Unterschied zum Beginn des 20. Jahrhunderts nicht daran beteiligt waren. ${ }^{2}$

Der 'Balkan' unterliegt folglich hochgradig Stereotypisierungen und Stigmatisierungen. Dabei ist die Fremdwahrnehmung, der Blick von außen entlarvend; eine Hauptrolle spielt die deutsche Literatur - im Unter-

1 Vgl. Peter Handke, Abschied des Träumers vom Neunten Land (1991), Frankfurt a. M. 1998, S. 16.

2 Vgl. Maria Todorova (Hg.), Balkan Identities. Nation and Memory, New York 2004, S. 9. 
schied zu anderen Literaturen. Dies mag im Wesentlichen zwei Gründe haben: Erstens berührt der deutsche Literaturraum das betroffene Gebiet direkt. Dabei spielt der historisch begründete Rückgriff auf das südöstliche Grenzgebiet der Habsburger Doppelmonarchie, welche spätestens mit der Annektierung von Bosnien und Herzegowina 1878 weit auf der Balkanhalbinsel vordrang, eine große Rolle. Zweitens formt sich Deutschlands außenpolitisches Selbstbewusstsein vornehmlich in Bezug auf das Gebiet des ehemaligen Jugoslawiens neu aus: Dies beginnt mit der vorzeitigen Anerkennung von Slowenien und Kroatien 1991 und kulminiert im eigenen deutschen Sektor im Protektorat Kosovo 1999.

Peter Handkes literarische Beiträge, die spätestens mit Eine winterliche Reise zu den Flüssen Donau, Save, Morawa und Drina oder Gerechtigkeit für Serbien anfangs 1996 ins Polemische umschlagen und entsprechend rezipiert werden, stehen in direktem Bezug mit den angeführten Gründen: Einerseits fühlt sich der Autor - wie auch beispielsweise seine Hauptfigur im Roman Die Wiederholung (1986), der IchErzähler Filip Kobal - Jugoslawien durch seine slowenische Herkunft verbunden; andererseits wehrt er sich gegen den offiziellen, durch die Medien vermittelten Wahrheitsanspruch in der Schuldzuweisung für die jüngsten Kriege auf dem Balkan. Dass ein literarisches Schwergewicht am Anfang einer solchen literarischen Diskurslinie einerseits von Nähe (biographische Herkunft) und andererseits von Distanzierung (gegenüber der offiziellen Deutschlandpolitik) steht, wirkt sich auf sämtliche deutschsprachigen Autorinnen und Autoren aus, die sich in der Folge mit dem Balkan auseinandersetzen. Mit dem behandelten Gegenstand wird aber immer auch ein bestimmter Modus des Erzählens verbunden. Charakteristisch ist die Funktion des Ich-Erzählers, auch wenn es einige deutschsprachige Autoren gibt, bei denen der jüngste Krieg in Ex-Jugoslawien als verdrängtes Moment durchdringt; dazu zählt die "englische Wallfahrt" Die Ringe des Saturn (1995) von W.G. Sebald oder der Roman Ohnehin (2004) von Doron Rabinovici. Der Balkanraum - so lautet meine These - figuriert in der deutschen Literatur größtenteils als Projektionsraum, der sich durchwegs auch auf eine historisch begründete Folie bezieht, aber der vor allem bestimmten Erzählverfahren unterliegt, von denen sich erst die jüngste Generation mit Saša Stanišić wieder löst.

Um den Erzählmodus bei Peter Handke zu eruieren, soll zunächst nur ein einziger Ausschnitt aus Eine winterliche Reise genauer untersucht werden, in dem sich die wechselnde Position des Ich-Erzählers als Taktik innerhalb einer größer angelegten Strategie entpuppt:

[...] [A]ls dann vom Frühjahr an die ersten Bilder [...] aus dem bosnischen Krieg gezeigt wurden, gab es einen Teil meiner selbst (immer wieder auch für "mein Ganzes" stehend), welcher die bewaffneten 
bosnischen Serben, ob Armee oder Einzeltöteriche [...] als "Feinde des Menschengeschlechts" empfand, in Abwandlung eines Worts von Hans Magnus Enzensberger $\mathrm{zu}$ dem irakischen Diktator Saddam Hussein $[\ldots] .^{3}$

Ein "Teil" des hier Erzählenden schlägt sich durchwegs auf die Seite des besetzten Sarajevos und übernimmt damit die offizielle Version der Medien. Nachdem dieser "Teil" sich sogar noch hinreißen lassen hatte, ein Todesurteil über "den bosnischen Serbenhäuptling Radovan Karadžić" auszusprechen, wird in der Parallelstelle immer noch innerhalb derselben Passage klar, warum er sich als "Teil meiner selbst" bezeichnet:

Und trotzdem, fast zugleich mit solchen ohnmächtigen Gewaltimpulsionen eines fernen Sehbeteiligten, wollte ein anderer Teil in mir (der freilich nie für mein Ganzes stand) diesem Krieg und diesen Kriegsberichterstattungen nicht trauen. Wollte nicht? Nein, konnte nicht. (Reise, S. 38)

Die Antithese des Zweifels an "diesem Krieg und diese[r] Kriegsberichterstattung" begründet sich nicht in einer anderen Sichtweise oder Perspektive, sondern in einer nochmaligen Spaltung des Subjekts: "ein anderer Teil in mir", der wiederum - wie im ersten Zitat - in einer Klammerbemerkung präzisiert wird. Das erste Mal in der Verurteilung der "Feinde des Menschengeschlechts" lautet sie: "immer wieder auch für 'mein Ganzes' stehend". Das zweite Mal folgt die Parenthese: "der freilich nie für mein Ganzes stand". Daraus müsste man logisch folgern, dass der Erzählende meistens und "immer wieder" hinter der offiziellen Version von Schuldzuschreibung an die "bewaffneten bosnischen Serben" steht. Doch nimmt man die zwei zitierten Stellen nochmals genauer unter die Lupe, so macht sich eine Divergenz zwischen Erzählposition und Modus der Aussage bemerkbar: Im ersten Abschnitt fällt das Hans Magnus Enzensberger zugeschriebene und das in der Klammer nicht weiter zugeschriebene Zitat auf. Gerade letzteres ist Ergebnis einer Erzählstrategie, welche zunächst im Modus Distanzierungen markiert und später auch begründet. Beim ersten "Teil meiner selbst" handelt es sich um einen "fernen Sehbeteiligten", dem das Nahe entgeht. In der Begründung, warum er der "Kriegsberichterstattung" nicht trauen "konnte", kehrt sich aber die Position endgültig:

3 Peter Handke, Eine winterliche Reise zu den Flüssen Donau, Save, Morawa und Drina oder Gerechtigkeit für Serbien, Frankfurt a. M., Suhrkamp 1996, S. 36/37. Fortan zitiert unter dem Kurztitel Reise. 
Allzu schnell nämlich waren für die sogenannte Weltöffentlichkeit auch in diesem Krieg die Rollen des Angreifers und des Angegriffenen, der reinen Opfer und der nackten Bösewichte, festgelegt und fixgeschrieben worden. Wie sollte, war gleich mein Gedanke gewesen, das nur wieder gut ausgehen, wieder so eine eigenmächtige Staatserhebung durch ein einzelnes Volk - wenn die serbokroatisch sprechenden, serbischstämmigen Muselmanen Bosniens denn nun ein Volk sein sollten - auf einem Gebiet, und das gleiche Recht!, hatten, und die sämtlichen drei Völkerschaften dazu kunterbunt, nicht bloß in der meinetwegen multikulturellen Hauptstadt, sondern von Dorf zu Dorf, und in den Dörfern selber von Haus zu Hütte, neben- und durcheinanderlebten? (Reise, S. 38/39)

Zwar entzieht sich der Erzählende damit der Rolle des einfachen Spießumkehrers, der sich aus grundlegenden Überlegungen gegen die offizielle Position der Medien stellt. Damit nämlich würde er genau in dieselbe Argumentationsstruktur fallen und nichts Neues ans Licht befördern. In einer weiteren Camouflierung der Position des Ich-Erzählers, in der Wiedererinnerung des darauf folgenden "Gedanken[s]", wird die Distanz zwischen Erzählzeit und erzählter Zeit hervorgehoben. Taktisch geschickt wird so ein Gedanke eingeführt, der zunächst die Bosniaken, das heißt die Moslems Bosniens, gleich den Serben zuschlägt. Zusätzlich gebraucht er den historisch diffamierenden Begriff des "Muselmanen", wie er uns aus Kinderliedern bekannt ist: "Sei doch kein Muselman, der das nicht lassen kann." $\mathrm{Zu}$ fragen wäre natürlich an dieser Stelle, in welchem Verhältnis die pejorativen Bezeichnungen, die immer wieder auftauchen, zueinander stehen. Ist nicht der "Einzeltöterich" eine Verharmlosung des Mörders, während "serbokroatisch sprechende, serbischstämmige Muselmanen" in dieser spezifischen Aufmischung den Bosniaken ihre Identität - ein wahrhaft explosiver Begriff in Ex-Jugoslawien abspricht? Die Frage bei Handke gebärdet sich als ein riesiges Aufbäumen und in der Folge als rhetorischer Kunstgriff, der in einer Exotisierung Bosniens an jener Stelle kulminiert, wo beschrieben wird, wie die drei "Völkerschaften dazu kunterbunt [...] von Haus zu Hütte, nebenund durcheinanderlebten". Im undifferenzierten Sammelsurium, in der Reduktion des Zivilisationsgrades und in der Betonung des Ungeordneten wird der Erzählende dem Balkan als Projektionsfläche westlicher Verdrängungen, Befürchtungen und heimlichen Wünschen vollauf gerecht. Durch die Diffamierung ("Muselmanen") und die Exotisierung erreicht der Text schließlich das, worauf die Gesamtstrategie hinausläuft - eine camouflierte Umkehr des Spießes: 
Und wie hätte wiederum ich mich verhalten, als ein Serbe dort in Bosnien, bei der, gelind gesagt, mir gar nicht entsprechenden Staates auf meinem, unserm, Gebiet? Wer nun war der Angreifer? (Reise, S. 39)

Was als Absicherung des Echten und Wahrhaften oder Nahen und Gerechten gilt, das Subjekt als Ich, wird hier erstmals in der Identifikation mit dem von den Medien als Täter gebrandmarkten Serben genannt. Später auf der Reise durch Serbien wird dieser Ich-Erzähler, der nicht mehr einer Spaltung unterliegt, auf "Realitätsembleme" (S. 55) hingewiesen und erfährt - ganz nach ethnologischem Muster bei der Entdeckung neuer Stämme und Gebiete - die Initiation als Ankommender "mit dem Aussprechen des zuvor auf der Straße eingelernten und [...] von der Verkäuferin auf der Stelle verstandenen Warenworts" (S. 56). Die Bemühung um das erzählende Subjekt im Text ist letztlich nur eine Taktik in einer größer angelegten Strategie, welche eine zu eindeutige Position einnimmt.

Dennoch hat Peter Handke damit einem Modus der Introspektion und der Fixierung auf einen Ich-Erzähler den Weg gebahnt, auf den sich die deutsche Literatur, die sich mit dem Balkan beschäftigt, weiterhin bezieht. Denn in der Folge entstehen meist von Balkan-Liebhabern, welche sich länger und auch noch nach dem Krieg in Bosnien aufhalten, unzählige journalartige Erzählungen. Über deren Qualität mag man unterschiedlicher Ansicht sein. Dennoch sprengen sie den Rahmen des journalistischen Alltagsgeschäfts und geben persönlichere Ansichten über diese Region wider. Dazu gehören zwei Kategorien: Zur ersten zählen allgemein gehaltene Abhandlungen, wobei Richard Wagners Der leere Himmel. Reise in das Innere des Balkan (2003) den umfassendsten Überblick verschafft. Der aus dem Banat stammende Deutsche ergänzt seine persönlichen Reiseerlebnisse im Balkan mit einem überaus reichen Wissen zur Region. In der Form essayistisch, im Anspruch wissenschaftlich, wird die Erzählposition selber nie explizit dargelegt.

Von ähnlicher Machart sind Bücher wie Balkan Transit (1998) des FAZ-Korrespondenten Matthias Rüb und Kroatien im Aufbruch. Ein Land zwischen Balkan und Europa des ehemaligen Schweizer Botschafters in Zagreb, Paul Widmer. Sie alle versuchen, höchst materialreiche Erklärungsmuster für die jüngsten Konflikte zusammenzutragen - meist über weite Strecken ohne theoretische Bezugnahme oder Unterfutterung. Sie erheben zwar auch keinen literarischen Anspruch, fallen aber gerade wegen ihres essayistischen Charakters in die erste Kategorie. In die zweite Kategorie fallen diejenigen Versuche, welche indirekt oder direkt literarischen Anspruch erheben. All diese Texte weisen eine Ich-Erzähler-Instanz auf und folgen damit Peter Handkes Modell. Erstes einschlägiges Beispiel dafür sind Sabine Riedels Portraits Ende der Ausgangs- 
sperre. Sarajevo nach dem Krieg (1997). Im letzten Essay dieser Sammlung mit der Überschrift "Steve, Georgina und ich oder Journalisten in Sarajevo" gibt die Autorin eine melancholische Innenansicht ins Reporterbusiness, Handkes Hauptangriffsziel. Diese Perspektive erlaubt es, die unterschiedlichen Kulturen der 'Internationalen' aufs Korn zu nehmen. So klar die Ich-Position der Erzählinstanz sein mag, so brüchig und schillernd gestalten sich in der Folge die Beobachtungen: Denn noch fremder als die Balkan-Kultur entpuppt sich die amerikanische. Der Prototyp Richard, ein Fotograf, gewissermaßen nach Bosnien verknurrt, schwärmt stetig von einem großen Künstler, der "surrealistisches Zeug" herstellt und davon leben kann, ohne journalistisch tätig sein zu müssen. Dieser schwatzt der Ich-Erzählerin den Kopf voll, während die Reporter die Vernichtung eines Waffenarsenals durch die NATO-Truppen verfolgen.

Ich hörte ihm zu, ich sah Hühner, wie sie mit ihren in der Sonne glänzenden Federn auf der Wiese herumstolzierten, zwischen den Schützenpanzern, auf denen Soldaten saßen, mit ihren kugelsicheren Westen, ihren Stahlhelmen, und ich dachte, daß diese Situation auch eine ziemlich starke surrealistische Qualität hatte. ${ }^{4}$

In dieser Reflexion zeigt sich ein Thema, das sich durch den ganzen Band zieht, besonders deutlich: Beginnt Kunst nicht eben da, wo wir es nicht vermuten? Als 'surrealistische' Marginalie? Auch ohne literarischen Anspruch erheben zu müssen, entsteht so bei Sabine Riedel ein kleines Stück Literatur. Ein kontrastierendes Gegenbeispiel eines Balkan-Essays bildet Inge M. Artl, die Herausgeberin des Dubrovnik-Bandes Europa erlesen. Durch die Aufnahme in diesen Sammelband ohne Vordruck impliziert die Autorin bereits literarischen Anspruch. Nicht nur in Bezug auf den französischen Präsidenten Mitterand gibt sie ihrer politischen Haltung deutlichen Ausdruck. Am Schluss hebt ihr Text von den eingangs gemachten Detailbeschreibungen in der Stadt Dubrovnik/Ragusa direkt nach dem Krieg völlig ab. Sie zieht mit der Bezeichnung "Schreckensherrschaft der Türken" und "Gauleiter des Großosmanischen Reiches" die Parallele zum Dritten Reich und malt den Teufel an die Wand:

Heute, kaum achtzig Jahre nach dem Ende der Türkenbarbarei auf dem Balkan, hat Ragusa schon wieder die Türken vor der Haustür, Truppen der auf ihre eigene Art faschistischen Türkei, die noch immer auf Hitlers Vorbild Atatürk schwört. Ausgerechnet diese Spezialisten für

4 Sabine Riedel, Ende der Ausgangssperre. Sarajevo nach dem Krieg, Frankfurt a. M. 1997, S. 125. 
Völkermord und ethnische Säuberungen - siehe Armenien, siehe Kurdistan - aus Kleinasien hat Europa sich als "Friedenstruppe" in ExJugoslawien geholt, gewiß die folgenschwerste all der politischen Fehlentscheidungen und moralischen Bankrotterklärungen, die es sich in diesem Bürgerkrieg geleistet hat. Als ob der hausgemachte Faschismus nicht genügen würde... ${ }^{5}$

Damit schließt dieser Essay. Natürlich hat Europa "Fehlentscheidungen" getroffen, ebenso steht noch die heutige Türkei in einer problematischen Tradition, von der sie sich immer noch nicht ganz gelöst hat. Doch erstens hat sich das Szenario einer türkischen Besetzung von Bosnien überhaupt nicht bewahrheitet und zweitens gebärdet sich die letzte Aussage gerade dadurch absolut, dass die Ich-Sprechinstanz in den Hintergrund tritt, und sie die Kulturgrenze zwischen 'Europa' einerseits und der Türkei andererseits beschwört. Damit wird exklusiv der Balkan zu Europa gezählt, die Zurückdrängung des "kranken Manns am Bosporus" wird weiterhin perpetuiert. Der Balkan scheidet offensichtlich immer noch die Geister, ob offen oder camoufliert. Dass diese Region aber auch als Übergang oder eigenständige Region wahrgenommen werden kann, zeigen erst die zwei jüngsten Romane, Die Stille ist ein Geräusch. Eine Fahrt durch Bosnien von Juli Zeh und Wie der Soldat das Grammofon repariert von Saša Stanišić.

Auch wenn wie bei Peter Handke Ich-Erzähler(in) und Autor(in) im Reisebericht scheinbar zusammenfallen, muss, schon um der Textstruktur willen, unterschieden werden zwischen erzählendem und erzähltem bzw. erlebendem Ich. Die Differenz (die bei Peter Handke sogar noch wie aufgezeigt - zu einer Spaltung derselben Instanz führen kann) ist wichtig, um das Verhältnis zur beschriebenen Welt genau aufzuzeigen, das in den bisherigen Texten statisch, in den folgenden hingegen dynamisch ist. So beginnt Juli Zeh mit einer intertextuellen Referenz auf Peter Handke, wenn sie die Ich-Erzählerin sprechen lässt:

Der Hund sitzt auf der Schwelle und fixiert mich. Ich versuche es noch einmal. "Vor etwa acht Jahren, als du noch klein warst, fragte mein Bruder einmal, wo die Städte Moslemenklavebihać und Belagertessarajevo liegen." Der Hund versteht nicht. "Ich will sehen, ob BosnienHerzegowina ein Ort ist, an den man fahren kann, oder ob es zusammen mit der Kriegsberichterstattung vom Erdboden verschwunden ist." Der Hund hört nicht zu. ${ }^{6}$

5 Inge M. Artl, Dubrovnik. Europa erlesen, Klagenfurt/Celovec 2001, S. 220.

6 Juli Zeh, Die Stille ist ein Geräusch. Eine Fahrt durch Bosnien, Frankfurt a. M. 2002, S. 11. Fortan zitiert unter dem Kurztitel Stille. 
In diesem Dialog mit dem stummen Gegenüber, dem noch unterstellt wird, dass es weder versteht noch zuhört, wird der Grund der "Fahrt durch Bosnien" dargelegt. Hier wird klar, dass - wie bei Handke - auch ein Beweis gegen die "Kriegsberichterstattung" geführt werden solle, was ein Leichtes sein wird, zumal Bosnien-Herzegowina existiert und nicht "vom Erdboden verschwunden ist". Damit führt der Text eine zweifache Brechung der Handke-Reminiszenz aus: Einerseits wird in der Aussage selbst die Sinnlosigkeit dieser Beweisführung klar, andererseits verstärkt die Inszenierung des stummen Gegenübers die Irrelevanz der Aussage nochmals. Das Schweigen des Hundes ist letztlich entlarvend, seine Begleitung der ganzen Reise eine fortwährende Relativierung der Position der Ich-Erzählerin. Die Teilung des Ichs wird so nicht mehr inszeniert, sondern externalisiert. Die Ich-Position an und für sich braucht sich auch nicht mehr festzulegen, sondern kann sich wandeln. Deutlich wird dies im Verhältnis zur muslimischen bosnischen Bevölkerung, der sie anfänglich sehr distanziert begegnet. Sie bezeichnet die Frauen als "Bosnierinnen" (S. 65), die Männer hingegen als "türkische Männer", die ihr unheimlich vorkommen, von denen sie, offensichtlich in einem Stundenhotel in Sarajevo "am Rand des türkischen Viertels" abgestiegen, bedrängt wird (S. 66). Die Fremde wird inszeniert. Das Türkische ist ihre Chiffre. Gegen Schluss des Buches, nach beinahe einem Monat in Bosnien, ringt sie sich durch, Srebrenica zu besuchen. Am Fluss Drina angekommen entwirft sie von ihm folgende Szenerie:

Der Fluss ist flach, man könnte zu Fuß nach Serbien laufen. In meiner Vorstellung war die Drina metertief und voll Salzsäure. In Višegrad wurde sie so lange mit verstümmelten Leichen gefüttert, bis der Betreiber des Wasserkraftwerks stromabwärts sich über verstopfte Turbinen beschwerte. Von vielen Augenzeugenberichten erwischte mich am heftigsten die Bemerkung, dass einige Leichen noch am Leben gewesen seien. Der Gedanke daran kommt hartnäckig immer wieder zurück, wie eine Wespe, während man auf der Terrasse eine Honigmelone verzehrt. Totschlagen kann man ihn nicht mit der flachen Hand, und wenn man ihn zu vertreiben versucht, wird er aggressiv. Nach dem letzten Körper wurde die Rote Drina wieder zu Jade und Türkis. Nichts wäscht sich schneller die Schmutzflecken ab als ein Fluss. Da drüben auf der anderen Seite stand Peter Handke vor fünfdreiviertel Jahren, entdeckte eine schwimmende Kindersandale und wollte nicht herüberkommen. Was haben sie ihn dafür gescholten. (Stille, S. 230/231)

In dieser Passage nimmt die Erzählfigur Bezug auf "Augenzeugenberichte", die sie beschäftigen und nicht loslassen. Gegenstand der Betrachtung ist die Drina, Grenzfluss zwischen Serbien und Bosnien. 
Bereits bevor Peter Handke namentlich erwähnt wird, nimmt sie in dieser Textstelle auf ihn Bezug, indem sie dem Fluss im Unterschied zu ihrem Vorgänger jegliche Faszination als Gegenstand abspricht: "Der Fluss ist flach". Vor ihrem inneren Auge jedoch bauen sich die furchtbaren Szenen auf; und sie gibt zudem zu bedenken, wie sehr die Oberfläche täuschen kann: "Nach dem letzten Krieg wurde die Rote Drina wieder zu Jade und Türkis." Diese Überzeichnung macht deutlich, wie sehr sie sich von der "Gegenständlichkeit" Handkes distanziert, bevor sie ihn dann auch - und hier das einzige Mal - namentlich erwähnt. Der letzte Satz, der als Knittelvers daherkommt ("Was haben sie ihn dafür gescholten."), stammt gleichsam aus einer anderen Zeit. Mit einer Scheinidentifizierung distanziert sich so die Erzählerfigur nochmals von ihrer Vorgabe. Als sie sich Srebrenica nähert, hat sie immer mehr Mühe den Ort auch wirklich zu finden: "Ich bin wütend, weil kein einziges Schild nach Srebrenica weist, weder lateinisch noch kyrillisch." (S. 231) Die Wut richtet sich nur auf den ersten Blick auf das Fehlende als vielmehr auf die serbischkyrillische Vereinnahmung in der Winterlichen Reise, wo der Erzähler in einem Busbahnhof auf alten Tafeln neben Tuzla und Beograd Srebrenica auf kyrillisch, "geradezu kalligraphisch" (S. 118) findet. So kämpft Juli Zehs Text an zahlreichen Stellen gegen die Winterliche Reise an, bis sie selbst in Srebrenica ankommt:

Wenigstens eines der internationalen Büros sollte ich aufsuchen. Wie viele Menschen leben hier und in welcher Zusammensetzung? Prügeln die Männer im Rausch ihre Frauen und womit? Liegt es am Kriegstrauma, am Alkohol, an beidem? Und wie klappt es mit den Rückkehrern? Die Consultants und Deputies würden auf Englisch vom Elend berichten und sich ausschweigen über das Gerücht, die Säuberung Srebrenicas sei abgesprochen gewesen zwischen Westmächten und Kriegsführern, weil die Moslemstadt mitten in serbischem Gebiet lag. Sonst wäre der Friedensvertrag nie unterzeichnet worden. [...] Ich habe keine Lust, die internationalen Organisationen aufzusuchen. Ich habe überhaupt keine Lust, mit jemandem zu reden. (Stille, S. 232/233)

Die Zweifel an einer offiziellen Version der "internationalen Organisationen" sind bei der Ich-Erzählerin ebenso groß wie beim gespaltenen Ich Peter Handkes. Doch das Ergebnis ist ein anderes. Das Fremde erscheint plötzlich nicht mehr so fremd wie eingangs, so dass sie auf der Rückkehr nach Tuzla Erleichterung verspürt, sobald sie die Republika Srpska verlässt:

Auf die erste Moschee reagiert mein Zwerchfell mit Freudenstichen. Nie hatte ich etwas mit Allah am Hut. Seit neuestem fühle ich mich besser in seiner Gegenwart. (Stille, S. 235) 
Die Ich-Erzählerin beschreibt sehr genau ihre Gefühlslage. Sie verbirgt nicht, dass sie eine Reise unternimmt, die auf der anderen Seite der Drina stattfindet und während der sie sich auch zusehends mit der Gegenseite politisch identifiziert. Die Polarisierung scheint zwar vorgegeben, doch gerade durch die Verwendung ironischer Versatzstücke setzt sie sich deutlich vom Ich-Erzähler in der Winterlichen Reise ab.

Noch einen Schritt weiter geht Saša Stanišić in seinem ersten Roman Wie der Soldat das Grammofon repariert: Die Hauptperson Aleksander wächst in Višegrad auf, just im Zentrum des Romans von Ivo Andrić, Die Brücke über die Drina (1945), worauf sich Peter Handke explizit und Juli Zeh implizit beziehen. Aus diesem Grenzort wurde die muslimische Bevölkerung mehrheitlich vertrieben. Aleksander, aus serbischmuslimischer Mischehe und in erster Linie ein überzeugter Tito-Anhänger wie sein Großvater, beschreibt aus Kinderperspektive, wie seine Familie zwischen die Fronten gerät und sie schließlich nach Deutschland fliehen müssen. Die Erzähltechnik zeichnet sich dadurch aus, dass sich das Ich weder teilt noch in eine Gefühlswelt zurückzieht, sondern vielmehr multipliziert. Die Erzählstruktur bleibt sich so konsequent gleich, doch die Person und damit die Fokalisation ändern sich ständig, weil die Anführungszeichen konsequent ausgelassen werden. Immer wenn einschneidende Erlebnisse berichtet werden, nimmt der oder die Betroffene die Position des Ich-Erzählers ein. Dies geschieht mit Zoran, der mit seinem Vater zu früh zurückkehrt und seine Mutter in flagranti mit dem Nachbarn erwischt (S. 63), mit Asija, die in Višegrad zurückbleibt und von Gewalt berichtet (S. 146-148), oder im Gedicht von Nena Fatima "was ich eigentlich will":

Ich will reden wieder reden

ich will reden wieder reden aber einen grund brauch ich

soll ein guter grund sein das ist so $[\ldots] .^{7}$

Der Auslöser für den Perspektivenwechsel innerhalb der Ich-Erzählung ist immer eine einschneidende Zäsur. In jeglicher Hinsicht bildet der Krieg die Hauptzäsur, welche auch den Roman in zwei ungleiche Hälften unterteilt: Im ersten längeren Teil werden die Kindheitserlebnisse von Alexander berichtet. Der Erzählfluss scheint noch nicht so sehr zu unterscheiden zwischen erlebendem und erzählendem Ich, was Herr Fazlagić, Alexanders Lehrer, in der persönlichen Ermahnung an den Betroffenen vor dem Aufsatzschreiben auf den Punkt bringt:

7 Saša Stanišić, Wie der Soldat das Grammofon repariert, München 2006. Fortan zitiert unter dem Kurztitel Soldat. 
Du hast dieses Jahr bei allen Aufsätzen das Thema verfehlt - zügle gefälligst deine Fantasie! Herr Fazlagić tritt an meinen Tisch und beugt sich zu mir hinunter. Und für die direkte Rede, sagt er und stützt sich mit den Fäusten auf die Tischplatte, gibt es Anführungszeichen, das weißt du, das brauche ich dir nicht jedes Mal zu erklären. (Soldat, S. 86)

In der großen Zäsur erscheint dann das Buch im Buch "Als alles gut war von Aleksander Krsmanović - Mit einem Vorwort von Oma Katarina und einen Aufsatz für Herrn Fazlagić" (S. 159). Der Aufsatz "Eine schöne Reise" enthält hier natürlich die obligaten Anführungszeichen. Doch genau hier wird die Beschreibung ad absurdum geführt, indem erzählendes und erlebendes Ich nicht mehr getrennt werden:

"Familie, wir fahren dieses Jahr na-ach...", rief letzte Woche mein Vater mit der Stimme eines enthusiastischen Fernsehmoderators und winkte mit den Hotelprospekten. "Ach, Papa, du sprichst doch nur, weil ich Herrn Fazlagić, Nicht-mehr-Genosse-Lehrer, beweisen soll, dass ich die Anführungsstriche beherrsche." "Ja, und außerdem spreche ich nie mit der Stimme eines enthusiastischen Fernsehmoderators." (Soldat, S. 173)

So greift das Ereignis in die Erzählung ein. Was hier humoristisch aufgefangen wird, thematisiert ein wesentliches Merkmal dieses Romans. Denn nach diesem Einschnitt beginnt der letzte Teil des Buches, in dem der Ich-Erzähler von Deutschland nach Bosnien geht, um Nachforschungen für seinen Roman über Hinterbliebene und Vermisste in seiner ehemaligen Heimat anzustellen. Die Erzählfunktion bekommt eine neue Qualität: Nicht nur rücken erlebendes und erzählendes Ich rein zeitlich näher zusammen, sondern erst hier wird durch den Kunstgriff offensichtlich, dass hinter dem erzählenden Ich des ersten Teils des Romans nicht einfach ein Kind steht - sondern eine weitere Vermittlungsinstanz, die sich bisher nur versteckt hielt: der im Jahre 2002, also zehn Jahre nach der Flucht aus Višegrad, zurückkehrende Aleksander. Die IchErzählinstanzen wechseln nicht nur von Person zu Person, sondern entpuppen erst während des Romans in der einen Hauptperson die verschiedenen zeitlichen Schichten. Das Erzählverfahren in diesem deutschen Roman geht von andern Vorlagen aus, in denen die suggerierte Authentizität zwischen Autor und Ich-Erzähler explizit übergangen wird: Als Beispiele wären hier die Geschichte einer im Krieg vergewaltigten Frau Als gäbe es mich nicht (1999) von Slavenka Drakulić oder der Roman in Form eines Tagebuchs Logiergäste (1995) der Kriegsjahre aus der Perspektive einer jungen Frau von Nenad Veličković. Diese bewusste Trennung geht wohl mit einem anderen Anspruch an die Literatur einher: Das 
Ich hat nicht nur (oder weniger) für Authentizität einzustehen, sondern verbürgt vielmehr für ein kollektives Gedächtnis, so schwierig dieser Begriff kulturell auch zu fassen ist.

Es ist erstaunlich, wie das authentisch wirken wollende und sich rückversichernde Ich dem deutschen (Reise-)Bericht über den Balkan inhärent ist. Der um sich selber kreisende Text versucht zwar im Hinterfragen offizieller Muster der "Kriegsberichterstattung" eine Eigenposition einzunehmen. Die polemische Rezeption der Winterlichen Reise spricht für sich: Entweder ist man für oder gegen das Balkanbild von Peter Handke. Dass manch andere literarischen Erzeugnisse der deutschen Literatur ins Fahrwasser dieser Polemik geraten, wird offensichtlich. Letztlich hat dies aber weniger mit dem Inhalt, beispielsweise mit der Grenzsituation des 'Balkan' (gerade auch als stigmatisierter Begriff) zu tun, sondern mit dem Erzählverfahren. Das Bestreben, authentisch zu wirken, desavouiert sich ziemlich schnell selber, wie das anhand von Inge M. Artl aufgezeigt werden konnte. Zwar versucht Peter Handke in einer beredten Sezierung des Ichs Authentizität zu schaffen. Dennoch gelingt es ihm letztlich nicht, eine unabhängige, wirklich reflektierte Haltung einzunehmen. Dieser Problematik weichen Reportagen aus, indem sie die personale Erzählfunktion gänzlich ausschalten. Zaghafte, aber spannende Versuche bilden Sabine Riedels Reportagen. Dort entsteht nicht in den allgemeinen Anschauungen, sondern in der personalisierten Gedankenwelt der 'Ich-Erzählerin' der Wendepunkt vom Stereotypischen weg. Während bei Juli Zeh Handkes Subtexte präsent sind, von denen sich die Autorin absetzt, indem das Ich eine Wandlung durchläuft und in den Dialog mit der fremden Kultur tritt, so beginnt der Dialog zwischen den Kulturen dank geschickt eingesetzter Erzählverfahren bei Saša Stanišić kreativ zu werden. Wahrscheinlich wird erst hier der stigmatisierte Begriff 'Balkan' entlastet. 